\title{
Pemphigus paranéoplasique associé à un lymphome folliculaire : à propos d'un cas
}

\author{
Salino $\mathrm{S}^{\mathbf{1}}$, Lachenal $\mathrm{F}^{\mathbf{1}}$, Amini $\mathbf{M}^{\mathbf{2}}$, Bodard $\mathrm{AG}^{\mathbf{1}}$, Biron $\mathbf{P}^{\mathbf{1}}$ \\ ${ }^{1}$ Centre anti-cancéreux Léon Bérard, Lyon, France \\ ${ }^{2}$ Hôpital de l'Hôtel Dieu, Lyon, France \\ salino@lyon.fnclcc.fr
}

Le pemphigus est une maladie bulleuse auto-immune rare touchant la peau et les muqueuses. La classification des pemphigus distingue trois principaux types : les pemphigus profonds, superficiels et paranéoplasiques (Martel et Joly 2001). Le pemphigus paranéoplasique a été individualisé et décrit pour la première fois en 1990 (Anhalt et al. 1990). Le diagnostic repose sur la coexistence de critères cliniques, histologiques et immunologiques ainsi que sur l'association à une néoplasie (hémopathie lymphoïde le plus souvent).

Le cas rapporté est celui d'une patiente de 52 ans, suivie depuis peu au Centre Léon Bérard (Lyon) pour une masse rétro-péritonéale, dont la biopsie a révélé qu'il s'agissait d'un lymphome folliculaire. Au moment de l'admission pour la première cure de chimiothérapie, la patiente a développé une éruption bulleuse cutanéo-muqueuse. A l'examen, de nombreuses érosions et quelques bulles (de 0.5 à $3 \mathrm{~cm}$ ) ont été retrouvées sur le tronc et des ulcérations invalidantes intéressant toute la face interne des joues, la face dorsale et les bords de la langue. Celles-ci rendaient l'élocution et l'alimentation difficiles. Il existait également des érosions avec un exsudat jaune-blanchâtre sur les lèvres ainsi qu'une conjonctivite. L'examen anatomopathologique et les analyses immunohistochimiques (immunofluorescences directe et indirecte) ont permis d'établir le diagnostic de pemphigus paranéoplasique.

Le traitement de l'hémopathie a reposé sur une chimiothérapie de type R-CHOP. Celle-ci a été associée à une corticothérapie par voie générale ainsi qu'à des soins locaux pour la prise en charge du pemphigus paranéoplasique. Les lésions cutanées ont rapidement disparu. En revanche, les ulcérations buccales sont restées importantes pendant plusieurs mois. Elles ont finalement régressé lentement et une diminution progressive de la dose de corticoïdes a pu être envisagée. Le lymphome folliculaire est actuellement en rémission. Il persiste une langue cicatricielle avec deux lésions légèrement érosives mais asymptomatiques sur les bords de la langue.

Le pemphigus paranéoplasique est une pathologie rare mais dont la survenue des symptômes est précoce. Les lésions endobuccales sont en général très invalidantes. Le pronostic vital du patient est souvent mis en jeu (Nousari et al. 1999). La rémission des lésions buccales n'est obtenue qu'après plusieurs mois de traitement. 